Fassini PF. Silent myocardial ischemia during ambulatory electrocardiographic monitoring in patients with effort angina. J Am Coll Cardiol 1983;3:934-9.

22. Mulcahy D, Keegan J, Crean P, Quyyumi A, Shapiro L, Wright C, Fox K. Silent myocardial ischaemia in chronic stable angina: a study of its frequency and characteristics in $150 \mathrm{pa}-$ tients. Br Heart J 1988;60:417-23.

23. Gunther H, Osterspey A, Tris-Muller I, Eggeling T, Hopp HW, Hilger $\mathrm{HH}$. The sensitivity of $24 \mathrm{~h}$ Holter monitoring and exercise testing for the recognition of myocardial ischemia: a comparative study. Eur Heart J 1988; (suppl N):46-9.

24. Cohn PF, Ventrovec GW, Nesto R, Gerber FR, Total Ischemia Awareness Program Investigators. The nifedipine-total ischemia awareness program: a nation survey of painful and painless myocardial ischemia including results of antiischemic therapy. Am J Cardiol 1989;63:534-9.

25. Tzivoni D, Gavish A, Benhorin J, Banai S, Keren A, Stern S. Day to-day variability of myocardial ischemic episodes in coronary artery disease. Am J Cardiol 1987;60:1003-5.

26. Campbell S, Barry J, Rocco MB, et al. Features of the exercise test that reflect the activity of ischemic heart disease out of hospital. Circulation 1986;74:72-80.
27. Quyyumi A, Crake T, Wright C, Mockus L, Fox K. The role of ambulatory ST-segment monitoring in the diagnosis of coronary artery disease: comparison with exercise testing and thallium scintigraphy. Eur Heart J 1987;8:124-9.

28. Rozanski A, Berman DS. The efficacy of cardiovascular nuclear medicine studies. Semin Nucl Med 1987;27:104-20.

29 Gibson RS, Watson DD, Craddock GB, Crampton RS, Kaiser DL, Denny MJ, Beller L. Prediction of cardiac events after uncomplicated myocardial infarction: a prospective study comparing predischarge exercise thallium-201 scintigraphy and coronary angiography. Circulation 1983;68:321-36.

30. Bairey CN, Rozanski A, Maddahi J, Resser KJ, Berman DS. Exercise thallium-201 scintigraphy and prognosis in patients with typical angina and negative exercise electrocardiography. Am J Cardiol 1989;64:282-7.

31. Quyyumi AA, Panza JA, Diodati JG, Dilsizian V, Fallahan TS, Bonow RO. Relation between left ventricular function at rest and with exercise and silent myocardial ischemia. J Am Coll Cardiol 1992;19:962-7.

\title{
Dobutamine stress echocardiography: Prevalence of a nonischemic response in a low-risk population
}

\begin{abstract}
The problems of population referral bias in the calculation of specificity in diagnostic testing for coronary artery disease have been previously described. Previous studies investigating the sensitivity and specificity of dobutamine stress echocardiography (DSE) have been subject to pretest and posttest referral biases, largely as a result of the requirement for coronary arteriography. This study determines the normalcy rate for DSE by examining a population at statistically low risk for coronary artery disease. The probability of significant coronary artery disease was determined for $\mathbf{8 2 8}$ consecutive patients referred for DSE at the University of Michigan, and groups were identified with $<10 \%$ and $<5 \%$ probability of disease. Four of 72 patients (5.6\%) with a normal baseline echocardiogram and a probability of coronary artery disease of $<10 \%$, and three of $38(7.9 \%)$ with a probability of $<5 \%$ were found to have an abnormal DSE, yielding normalcy rates of $94.4 \%$ and $92.1 \%$, respectively. The area of abnormality involved the posterior circulation in three of four patients $(75 \%)$. This study demonstrates that DSE has a normalcy rate of $92 \%$ to $94 \%$ and is an accurate test for excluding the presence of significant coronary artery disease. (AM HEART J 1993;125:1257.)
\end{abstract}

David S. Bach, MD, Anne Hepner, MD, Pamela A. Marcovitz, MD, and William F. Armstrong, MD Ann Arbor, Mich.

Dobutamine stress echocardiography (DSE) is an accurate and feasible method for the noninvasive de-

From the Division of Cardiology, Department of Internal Medicine, University of Michigan Medical Center.

Received for publication Sept. 21, 1992; accepted Nov. 6, 1992.

Reprint requests: David S. Bach, MD, University of Michigan Hospital, UH B1F245-Box 0022, 1500 E. Medical Center Dr., Ann Arbor, MI 48109.

Copyright 1993 by Mosby-Year Book, Inc.

$0002-8703 / 93 / \$ 1.00+.10 \quad \mathbf{4} / \mathbf{1} / \mathbf{4 4 9 2 2}$ tection of coronary artery disease. ${ }^{1-10}$ Using selective coronary angiography as a measure of accuracy, the sensitivity of DSE for the detection of coronary artery disease has been high, ranging from $68 \%$ to $97 \% .^{3-10}$ The specificity of DSE, however, has been more variable, ranging from as high as $100 \%^{4}$ to as low as $45 \% .^{3}$ Several factors may be responsible for the low test specificity in these studies. In addition to the possibility of error in test interpretation, pretest 
and posttest selection biases may have been introduced in the selection of study populations. These biases exist to a large extent because of the requirement of invasive coronary arteriography for the documentation of coronary artery disease, and have been noted previously to influence the specificity of examinations in "unselected" patient populations. ${ }^{11}$

Several studies have addressed the problems of specificity calculation for diagnostic testing in the exclusion of coronary artery disease ${ }^{12-15}$ Pretest selection bias occurs ${ }^{16,17}$ when the study population is made up of patients with a high pretest probability of disease. In the case of DSE, factors contributing to this include the conduction of studies in the setting of university-based tertiary referral hospitals where the incidence and severity of coronary artery disease is greater than in the general population, and the inclusion of patients in the study population who already have the established diagnosis of coronary artery disease. This includes patients undergoing testing following myocardial infarction, and patients referred for DSE following cardiac catheterization for the assessment of the significance of already detected coronary artery disease.

Inclusion of patients with nonischemic cardiac disease may also contribute to pretest referral bias. These patients often have wall motion abnormalities at rest and thus are more likely to have false positive DSEs than are patients without underlying cardiac diseases. ${ }^{3}$ Additionally, they are more likely to undergo diagnostic cardiac catheterization. As such, the pool of patients undergoing cardiac catheterization includes a disproportionate number of patients preselected to have a false positive test.

Posttest referral bias may also contribute to a poor specificity for DSE. As the test has gained clinical use since its introduction, patients are more likely to undergo subsequent diagnostic cardiac catheterization following a positive DSE than following a negative test. As such, most patients with a false positive test will undergo coronary arteriography, while the majority of patients with negative results will not. As has been previously described, ${ }^{16}$ this will result in a decline in the measured test specificity.

These referral biases may be responsible for the relatively poor test specificity of DSE in previous studies. Given these constraints on population selection, alternative means must be used for determining the ability of the test to accurately predict the absence of disease. Precedent exists in clinical studies for the use of normalcy, the occurrence of a negative test in a population at statistically low risk for disease, ${ }^{18 .} 19$ to decrease the selection bias accompanying the requirement for invasive coronary angiog- raphy. This study was designed to assess the normalcy rate of DSE by reviewing tests performed in patients who are at statistically low risk of having significant coronary artery disease.

\section{METHODS}

Study population. Clinical characteristics and DSE results were retrospectively reviewed for all patients referred for DSE at the University of Michigan Medical Center between January 1990 and September 1991. The probability of significant coronary artery disease was established using a previously described nomogram. ${ }^{20}$ Probability was based on the age and sex of the patient, a history of diabetes, hyperlipidemia or tobacco use, previous history of myocardial infarction or abnormalities on electrocardiography, and a history of typical or atypical chest pain. Based on these criteria, patients were identified with a probability of significant coronary artery disease of less than $10 \%$. Additionally, subsets of patients with a probability of coronary artery disease of less than $55^{\prime \prime}$, and those with a probability of disease between $5 \%$ and $10 \%$ were identified.

Dobutamine echocardiography. Dobutamine stress echocardiograms were performed using a previously reported protocol. ${ }^{10}$ The protocol was undertaken after approval of the University of Michigan Human Subjects Committee, and informed consent was obtained from each patient. Dobutamine was administered as a continuous intravenous infusion in 3-minute stages at doses of $5,10,20$, and $30 \mu \mathrm{g} / \mathrm{kg} / \mathrm{min}$. Heart rate was continuously monitored. Images were obtained in standard parasternal long- and short-axis views, and in apical four-chamber and twochamber views. Two-dimensional echocardiograms were recorded at baseline and at each stage of dobutamine infusion. Blood pressure and 12-lead electrocardiography were obtained at baseline, at the completion of each stage, and at 5 minutes following infusion termination. Indications for discontinuation of the protocol included the development of significant chest pain or a new wall motion abnormality on echocardiography. Echocardiographic images were digitized at baseline, during $5 \mu \mathrm{g} / \mathrm{kg} / \mathrm{min}$ infusion, at peak dobutamine infusion, and at 5-minutes of recovery.

All analyses were completed by a highly experienced observer (WFA). Echocardiograms were interpreted prospectively, intermixed with other daily stress and dobutamine stress studies, and nomogram-derived probabilities of coronary artery disease were subsequently calculated. As such, the interpreter was blinded to the nomogram-derived probability of coronary artery disease at the time of interpretation of the echocardiogram.

A normal response to dobutamine infusion is hyperdynamic motion in all visualized areas of the left ventricle. An abnormal test for the detection of coronary artery disease was defined as the presence of a resting wall motion abnormality, or the induction of a new or worsened wall motion abnormality during dobutamine infusion. The latter was considered as evidence of inducible ischemia.

Catheterization. Cardiac catheterization was performed between 0 and 149 (mean $47.5 \pm 61.3$, median 8 ) days of the DSE in nine patients in whom there was a clinical indica- 
Table I. Patient demographics

\begin{tabular}{|c|c|c|c|c|c|c|c|c|c|c|c|}
\hline $\begin{array}{c}C A D \text { prob- } \\
\text { ability }\end{array}$ & $\begin{array}{l}\text { Population } \\
\text { size }(n)\end{array}$ & $\begin{array}{l}\text { Age } \\
\text { range } \\
(y r)\end{array}$ & $\begin{array}{c}\text { Age } \\
\text { mean } \\
(y r)\end{array}$ & $\begin{array}{c}\text { Age } \\
\text { median } \\
(y r)\end{array}$ & $\begin{array}{l}\text { No. } \\
\text { male }\end{array}$ & $\begin{array}{c}\text { No. } \\
\text { female }\end{array}$ & $\begin{array}{l}\text { Atypical } \\
\text { chest } \\
\text { pain }\end{array}$ & $\begin{array}{l}\text { Diabetes } \\
\text { mellitus }\end{array}$ & $\begin{array}{c}\text { Hypercholes- } \\
\text { terolemia }\end{array}$ & $\begin{array}{c}\text { Tobacco } \\
\text { use }\end{array}$ & $\begin{array}{c}S T \text { segment } \\
T \text { wave } \\
\text { abnormalities }\end{array}$ \\
\hline$<10^{\circ}$ & 75 & $19-65$ & 41.8 & 41 & 20 & 55 & 37 & 4 & 1 & 4 & 3 \\
\hline$<5 C_{i}^{\circ}$ & 39 & $19-52$ & 37.5 & 37 & 9 & 30 & 13 & 1 & 1 & 1 & 1 \\
\hline
\end{tabular}

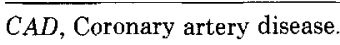

Table II. Normalcy rate of dobutamine stress echocardiography (DSE)

\begin{tabular}{|c|c|c|c|c|c|c|}
\hline \multirow{2}{*}{$\begin{array}{c}C A D \\
\text { prob- } \\
\text { ability }\end{array}$} & \multicolumn{2}{|c|}{ All subjects } & \multicolumn{2}{|c|}{ Normal baseline echocardiogram } & \multirow[b]{2}{*}{$\%$} & \multirow[b]{2}{*}{ Normalcy } \\
\hline & $\begin{array}{l}\text { Population } \\
\text { size }(n)\end{array}$ & $\begin{array}{c}\text { Abnormal } \\
\text { DSE }\end{array}$ & $\begin{array}{c}\text { Population } \\
\text { size (n) }\end{array}$ & $\begin{array}{c}\text { Abnormal } \\
\text { DSE }\end{array}$ & & \\
\hline$<10 \%$ & 75 & 5 & 72 & 4 & 5.6 & $94 \%$ \\
\hline$<5 \%$ & 39 & $4^{*}$ & 38 & $3 \dagger$ & 7.9 & $92 \%$ \\
\hline $5 \%-10 \%$ & 36 & $1^{*}$ & 34 & $1 \uparrow$ & 2.9 & $97: 0$ \\
\hline
\end{tabular}

Abbreviations as in Table I.

${ }^{*} p=0.36$.

$\dagger p=0.62$.

tion for the procedure. The angiograms were reviewed and interpreted by an experienced observer. Significant obstructive coronary artery disease was felt to be present if there was a $50 \%$ or greater stenosis in any of the major epicardial coronary arteries.

Statistical analysis. The incidence a of positive DSE for the groups with probability of coronary artery disease of less than $5 \%$ and for those with probability of disease of $5 \%$ to $10 \%$ were compared using a Fisher exact test (two-tail). The Fisher exact test was also used for comparison of the incidence of a positive DSE among male and female patients. A significant difference was defined as $p<0.05$.

\section{RESULTS}

Patient demographics. Clinical characteristics of 828 patients referred for DSE were reviewed. Of these, 75 patients were identified with a probability of significant coronary artery disease of less than $10 \%$. This group included $20(27 \%)$ men and 55 $(73 \%)$ women. The age range was 19 to 65 years, with a mean of 41.8 years. The age range of the male patients was 19 to 45 (mean $33.1 \pm 5.1$ ) years, and that of the female patients was 26 to 65 (mean $44.8 \pm 9.6$ ) years. Clinical characteristics are summarized in Table I.

Indications for referral for DSE included evaluation of chest pain in 30 patients, preoperative cardiac risk assessment in 23 (including eight patients undergoing evaluation for lung transplantation), evaluation of palpitations or arrhythmia in seven, evaluation of dyspnea or conduction abnormality in two each, evaluation of syncope or dilated cardiomyopathy in one patient each, and completion of other protocols in six patients. No indication was available in three patients.

Thirty-seven patients $(49 \%)$ had a history of atypical chest pain. Only 11 patients $(15 \%)$ had one or more risk factors that increased the probability of coronary artery disease, using the nomogram of Pryor et al. ${ }^{20}$ These risk factors included diabetes in four patients, hyperlipidemia in one patient, a history of tobacco use in four patients, and baseline ST segment or $\mathrm{T}$ wave abnormalities on resting electrocardiography in three patients. A single patient had two risk factors; a 30-year-old man with diabetes and resting ST segment abnormalities on electrocardiography, who was referred for DSE as part of a preoperative cardiac evaluation. No patients had a history of previous myocardial infarction or electrocardiographic evidence of a previous myocardial infarction. Thirtynine patients had a probability of coronary artery disease of less than $5 \%$.

Dobutamine stress echocardiography. The DSE protocol was completed in all 75 patients. No patients developed chest pain during the infusion. Among all patients with a probability of coronary artery disease of less than $10 \%$, DSE was positive for ischemia in five patients $(6.7 \%)$, including four $(80 \%)$ women. These data are summarized in Table II. The incidence was $7.2 \%$ ( 4 of 55 ) in women and $5.0 \%$ ( 1 of 20 ) in men $(p=1.0)$. The age range for these patients was 31 to 52 (mean $41.4 \pm 8.8$ ) years. One patient, whose status was evaluated 4 days after pericardiocentesis, had a resting inferior wall motion abnormality and a small residual pericardial effusion. None of the other 
Table III. Abnormal dobutamine stress echocardiograms (DSEs)

\begin{tabular}{|c|c|c|c|c|c|c|c|c|c|}
\hline $\begin{array}{l}\text { Age } \\
(y r)\end{array}$ & Sex & $\begin{array}{c}\text { Probability } \\
\text { of CAD }\end{array}$ & $\begin{array}{c}D S E \\
\text { indication }\end{array}$ & $\begin{array}{c}\text { CAD } \\
\text { rish } \\
\text { factors }\end{array}$ & $\begin{array}{c}\text { Baseline } \\
\text { echocardiogram }\end{array}$ & $\begin{array}{c}\text { Induced } \\
\text { wall motion } \\
\text { abnormality }\end{array}$ & $\begin{array}{l}\text { Abnormal } \\
\text { circulation }\end{array}$ & $\begin{array}{l}\text { Coronary } \\
\text { angiogram }\end{array}$ & Other \\
\hline 31 & $\mathbf{M}$ & $<5^{c}$ & Atyp $\mathrm{CP}$ & None & Normal & Inferior & Posterior & $\mathrm{N} / \mathrm{A}$ & \\
\hline 34 & $\mathrm{~F}$ & $<5$ & Preop & Tobacco & Normal & Inferior & Posterior & Normal & \\
\hline 43 & F & $<5 \%$ & Atyp CP & None & Normal & Inferior & Posterior & Normal & \\
\hline 47 & F & $<5^{\circ}$ & Preop & $\mathrm{ST}$ abn & $\begin{array}{r}\text { Inferior } \\
\text { WMA }\end{array}$ & Septal & Anterior & $\mathrm{N} / \mathrm{A}$ & PEF* \\
\hline 52 & $\mathbf{F}$ & $<10^{\circ}$ & Atyp CP & None & Normal & $\begin{array}{l}\text { Infero- } \\
\text { posterior }\end{array}$ & Posterior & Normal & \\
\hline
\end{tabular}

Atyp CP, Atypical chest pain; Preop, preoperative assessment; ST abn, ST segment abnormalities; WMA, wall motion abnormality; N/A, not available; other abbreviations as in Table I.

*Residual pericardial effusion 4 days following pericardiocentesis.

four patients had a resting wall motion abnormality. The area of induced wall motion abnormality was anterior in one patient, inferior in three, and inferoposterior in one. Three of these five patients underwent coronary arteriography. None of these three patients, nor any of the other six patients with negative DSEs undergoing coronary arteriography had evidence of significant coronary artery disease. Four of 39 patients $(10.2 \%)$ with a less than $5 \%$ probability of coronary artery disease, and one of 36 patients $(2.8 \%)$ with a probability of coronary artery disease between $5 \%$ and $10 \%$ had an abnormal DSE (T'able II). Induced wall motion abnormalities involved the inferior wall in three and the anterior wall in one patient. The clinical and echocardiographic characteristics of these patients are summarized in Table III.

In addition to the patient with residual pericardial effusion and a baseline inferior wall motion abnormality 4 days following pericardiocentesis, two patients in the group had a severe dilated cardiomyopathy. Excluding patients with underlying cardiomyopathy, pericardial or valvular disease, 3 of 38 patients $(7.9 \%)$ with a probability of coronary artery disease of less than $5 \%$, and 1 of 34 patients $(2.9 \%)$ with a probability of disease between $5 \%$ and $10 \%$ had a DSE positive for ischemia. There was no statistically significant difference between these groups with respect to the incidence of an abnurmal DSE $(p=0.62)$. In the group of 72 patients with a probability of coronary artery disease of less than $10 \%$ and a normal baseline echocardiogram, four patients $(5.6 \%)$ had an abnormal DSE.

\section{DISCUSSION}

Previous studies assessing the accuracy of DSE for the detection of coronary artery disease have been performed using patient populations subject to pretest and posttest referral bias. Study populations have been drawn from university-based settings and have included patients following acute myocardial infarction, patients referred from the cardiac catheterization laboratory, and patients referred for cardiac catheterization based on the results of the DSE. In each case, either pretest selection bias existed such that the population under study had a greater prevalence of coronary artery disease than would be expected in a general population referred to the echocardiography laboratory, or posttest referral bias existed as a result of referral for subsequent cardiac catheterization based on the results of the echocardiogram. Because of these selection biases, the study populations may have contained fewer than expected numbers of patients without coronary artery disease, and determination of the test specificity may have been subsequently impaired. To circumvent these selection biases, this study was designed to assess the ability of DSE to exclude the presence of significant coronary artery disease in a population at very low risk for disease. As risk for coronary artery disease was used for selection criteria, none of the biases in patient selection that accompany studies requiring cardiac catheterization as a comparative standard were operative. Clinical characteristics were reviewed for patients referred for DSE, and patients with a less than $10 \%$ or a less than $5 \%$ probability of having significant coronary artery disease were identified using a previously described nomogram for the prediction of coronary artery disease probability in an individual. Particularly in the lowest probability group, the patient population was skewed to contain a predominance of women and patients under 40 to 50 years of age, as well as patients without significant independent risk factors for coronary artery disease. The patients were otherwise representative of patients referred for DSE.

The incidence of an abnormal and presumed false 
positive DSE was $6.7 \%$ (5 of 75 patients). Excluding patients who had baseline cardiomyopathy, pericardial or valvular disease, the incidence was $5.6 \%$. The incidence was $7.9 \%$ among those with a probability of coronary artery disease of less than $5 \%$, and $5.6 \%$ in the group of patients with a probability of less than $10 \%$, yielding normalcy rates for DSE of $92 \%$ and $94 \%$, respectively. A higher incidence of an abnormal DSE was noted in the group with a lower predicted coronary disease probability, but the difference is not statistically significant and is most likely a function of the relatively small size of the two groups. The normalcy rate of $92 \%$ to $94 \%$ is in close agreement with the specificity of $91 \%$ for DSE in patients with normal baseline echocardiograms previously reported from our laboratory. ${ }^{10}$ This population of patients with normal resting function excludes many patients with nonischemic cardiac discases, and results in fewer false positive tests.

The majority of false positive DSEs in this study were in the posterior circulation, with only one patient developing an inducible abnormality in the anterior circulation. This is consistent with findings in previous studies that inducible abnormalities in the posterior circulation are more likely to represent false positive DSEs than do anterior circulation abnormalities. ${ }^{10}$ The mechanism underlying this observation is uncertain, but many represent either observer error because of suboptimal visualization in this area, or wall motion abnormalities related to nonischemic but as yet unidentified mechanisms.

Conclusions. DSE has been previously demonstrated to be highly sensitive for the detection and localization of coronary artery disease. Test specificities ranging from $100 \%$ to $45 \%$ in previous studies may have been compromised by potential pretest and posttest referral biases in the populations studied. This study demonstrates that DSE has a normalcy rate of $92 \%$ to $94 \%$ in patients at low likelihood of having disease, with false positive tests predominantly involving the posterior circulation. DSE is a reasonable test for excluding the presence of significant coronary artery disease in nonselected patient populations.

\section{REFERENCES}

1. Berthe C, Pierard LA, Hiernaux M, Trotteur G, Lempereur $P$, Carlier J, Kulbertus HE. Predicting the extent and location of coronary artery disease in acute myocardial infarction by echocardiography during dobutamine infusion. Am J Cardiol 1986;58:1167-72.

2. Mannering D, Cripps T, Leech G, Mehta N, Valantine H, Gil- more $\mathrm{S}$, Bennett ED. The dobutamine stress test as an alternative to exercise testing after acute myocardial infarction. $\mathrm{Br}$ Heart J 1988;59:521-6.

3. Marcovitz PA, Mathias W, Dick RJL, Armstrong WF. Detection of regional coronary stenoses in patients with complex coronary anatomy using dobutamine stress echocardiography [Abstract]. .J Am Coll Cardiol 1991;17:278A.

4. Sawada SG, Segar DS, Ryan T, Brown SE, Dohan AM, Williams R, Fineberg NS, Armstrong WF, Feigenbaum H. Echocardiographic detection of coronary artery disease during dobutamine infusion. Circulation 1991;83:1605-14.

5. Previtali M, Lanzarini L, Ferrario M, Tortorici M, Mussini A, Montemartini C. Dobutamine versus dipyridamole echocardiography in coronary artery disease. Circulation 1991;83(suppl III):III27-31.

6. Cohen JL, Greene TO, Ottenweller J, Binenbaum SZ, Wilchfort SD, Kim CS. Dobutamine digital echocardiography for detecting coronary artery disease. Am J Cardiol 1991; $67: 1311-8$.

7. Martin TW, Seaworth JF, Johns JP, Pupa LE, Condos WR. Comparison of adenosine, dipyridamole, and dobutamine in stress echocardiography. Ann Intern Med 1992;116:190-6.

8. Mazeika PK, Nadazdin A, Oakley CM. Dobutamine stress echocardiography for detection and assessment of coronary artery disease. J Am Coll Cardiol 1992;19:1203-11.

9. Segar DS, Brown SE, Sawada SG, Ryan T, Feigenbaum H. Dobutamine stress echocardiography: correlation with coronary lesion severity as determined by quantitative angiography. J Am Coll Cardiol 1992;19:1197-202.

10. Marcovitz PA, Armstrong WF. Accuracy of dobutamine stress echocardiography in detecting coronary artery disease. Am J Cardiol 1992;69:1269.73.

11. Ransohoff DF, Feinstein AR. Problems of spectrum and bias in evaluating the efficacy of diagnostic tests. $N$ Engl $J$ Med 1978;299:926-30.

12. Philbrick JT, Horwitz RI, Feinstein AR. Methodologic problems of exercise testing for coronary artery disease: groups, analysis and bias. Am J Cardiol 1980;46:807-12.

13. Philbrick JT, Horwitz RI, Feinstein AR, Langou RA, Chandler JP. The limited spectrum of patients studied in exercise test research. Analyzing the tip of the iceberg. JAMA 1982; 248:2467-70.

14. Rozanski A, Diamond GA, Forrester JS, Berman DS, Morris D, Swan HJC. Alternative referent standards for cardiac normality. Implications for diagnostic testing. Ann Intern Med 1984;101:164-71.

15. Iskandrian AS, Heo J. Appraisal of false positive results in nuclear cardiac imaging. AM HEART J 1990;119:708-9.

16. Rozanski A, Diamond GA, Berman D, Forrester JS, Morris D, Swan HJC. The declining specificity of exercise radionuclide ventriculography. N Engl J Med 1983;309:518-22.

17. Detrano R, Janosi A, Lyons KP, Marcondes G, Abbassi N, Froelicher VF. Factors affecting sensitivity and specificity of a diagnostic test: the exercise thallium scintigram. $\Lambda \mathrm{m} J$ Med 1988;84:699-710.

18. Diamond GA, Rozanski A, Forrester JS, Morris D, Pollock BH, Staniloff HM, Berman DS, Swan HJC. A model for assessing the sensitivity and specificity of tests subject to selection bias. Application to exercise radionuclide ventriculography for diagnosis of coronary artery disease. J Chronic Dis 1986;39:343-55.

19. Marwick TH, Nemec JJ, Pashkow FJ, Stewart WJ, Salcedo EE. Accuracy and limitations of exercise echocardiography in a routine clinical setting. J Am Coll Cardiol 1992;19:74-81.

20. Pryor DB, Harrell FE Jr, Lee KL, Califf RM, Rosati RA. Estimating the likelihood of significant coronary artery disease. Am J Med 1983;75:771-80. 